Article

\title{
Frequency-Selective Wallpaper for Indoor Interference Reduction and MIMO Capacity Improvement
}

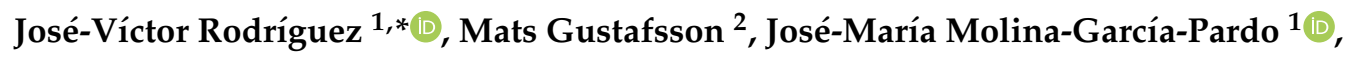 \\ Leandro Juan-Llácer ${ }^{1}(\mathbb{D})$ and Ignacio Rodríguez-Rodríguez ${ }^{3}(\mathbb{D}$ \\ 1 Departamento de Tecnologías de la Información y las Comunicaciones, Universidad Politécnica de \\ Cartagena, E30202 Cartagena, Spain; josemaria.molina@upct.es (J.-M.M.-G.-P.); leandro.juan@upct.es (L.J.-L.) \\ 2 Department of Electrical and Information Technology, Lund University, Box 118, SE-221 00 Lund, Sweden; \\ mats.gustafsson@eit.lth.se \\ 3 Departamento de Ingeniería de Comunicaciones, ATIC Research Group, Universidad de Málaga, \\ E29071 Málaga, Spain; ignacio.rodriguez@ic.uma.es \\ * Correspondence: jvictor.rodriguez@upct.es
}

Received: 28 January 2020; Accepted: 10 April 2020; Published: 1 May 2020

check for updates

\begin{abstract}
This paper presents the design and features of frequency-selective wallpaper-based on periodic and symmetric metallic hexagons-intended to be attached to standard walls for filtering out $5 \mathrm{GHz}$ signals (e.g., IEEE 802.11a systems) without blocking other selected radio communication services (e.g., cellular mobile communication signals). It analyzes the characteristics of the radio channel-as found within standard indoor environments-with both regular walls and walls with the proposed frequency-selective wallpaper, examined using a ray-launching program for single-input single-output (SISO) and multiple-input multiple-output (MIMO) systems. This allows the harvesting of parameters, including channel capacity, power delay profile, and signal-to-interference ratio, for proper comparison between the two environments under study: with and without the presented wallpaper. The achieved results clearly show that the use of the proposed frequency-selective wallpaper in an indoor scenario reduces interference levels by an additional attenuation of up to $20 \mathrm{~dB}$ in comparison to an unpapered wall. Additionally, with MIMO systems, radio channel characteristics, such as capacity, are improved due to the increase in the magnitude of all singular values of the channel transfer matrix compared to the unpapered wall case, thereby leading to the existence of more relevant subchannels.
\end{abstract}

Keywords: frequency-selective surfaces; radio channel architecture; MIMO systems

\section{Introduction}

Over the last 10 years, the increase in wireless communication systems has made it necessary to minimize the interference between different systems in the same environment, and to resolve difficulties of capacity caused by strains imposed by user numbers and spectrum availability. Techniques proposed for mitigating these problems have included new antenna designs and advanced signal processing technology. Unfortunately, these measures are frequently complicated and/or costly. An alternative means of addressing this problem in indoor environments would be modification of the physical wave propagation environment by applying frequency-selective (F-S) wallpaper to the walls to block (reflect) unwanted interference while still allowing the desired radio communication services to pass through [1-7]. Such a strategy can improve the signal-to-interference ratio (SIR) [8,9].

Because F-S wallpapers restrict the frequency signal of the systems that are being blocked to the room where they are located, radio channel characteristics can experience improvement using high-performance systems, e.g., multiple-input multiple-output (MIMO) systems, that usually gain within rich scattering environments and where radio channel capacities are expanded by the quantity of 
uncorrelated channels and the strength of each [10]. Therefore, this paper, extending the analysis carried out in previous studies where the performance solely of single-input single-output (SISO) systems was examined in indoor environments with F-S surfaces [1-9,11], presents the design and features of new F-S wallpaper when both SISO and MIMO systems are considered. The wallpaper-based on periodic and symmetric metallic hexagons-is intended to be attached to standard walls for filtering out $5 \mathrm{GHz}$ signals (e.g., IEEE 802.11a systems) without blocking other selected radio communication services (e.g., cellular mobile communication signals). This way, the presented work analyzes the characteristics of the radio channel—as found within standard indoor environments—with both regular walls and walls with the proposed F-S wallpaper, examined using a ray-launching program. Thus, the capacity for the proposed F-S wallpaper to effect improvements in SIR and to achieve improved radio channel characteristics for MIMO systems is evaluated, while at the same time analyzing the singular values for the channel transfer matrix.

\section{Wallpaper Design}

\subsection{Frequency-Selective Surfaces}

Numerous antenna systems for modern fixed and mobile communications services rely on F-S surfaces (FSSs). FSSs effectively comprise array structures consisting of many thin conducting elements, frequently supported by being printed on dielectric substrate. They act as passive electromagnetic filters. Such arrays often act as periodic apertures within the conducting plane. If an FSS comprises a conductor array, reflection occurs at the surface of incident waves for particular frequencies (stop band or reflection band); at other frequencies the service allows these waves to pass through (pass-band or transmission band). With conducting arrays, these resonances occur because of high-induced element currents. This makes the surface act as a metallic sheet at certain resonances. If an aperture array is employed, the majority of the FSS is reflective, exhibiting a pass-band as a resonance resulting from strong fields within apertures [12,13].

\subsection{Wallpaper with Low Transmission at $5 \mathrm{GHz}$}

The wallpaper was designed using the software Periodic Boundary Finite-difference Time-domain (PB-FDTD), centered on unit cell analysis techniques with a baseline numerical technique being the FDTD methodology. This program employs periodic boundary conditions for the reduction of computational volumes to single-unit cells. In the unit cell technique, the number of possible scan angles is a discrete number, which is determined by the number of FDTD cells in the unit cell (it should be noted that the FDTD mesh needs to be periodic). Such a number of possible scan angles increases with the number of FDTD cells. Then, the elements of the array are excited with broadband Gaussian pulses with appropriate time delays to scan the array in the desired direction. This way, the simulation continues until the field at a test point of the FDTD volume has decreased within a specific factor. The main goal of such wallpaper was to block or confine $5 \mathrm{GHz}$ signals (for example IEEE 802.11a systems), while simultaneously permitting wireless services on different frequencies (for example, cellular mobile communication signals) access. For this purpose, we considered the FSS geometry illustrated in Figure 1.

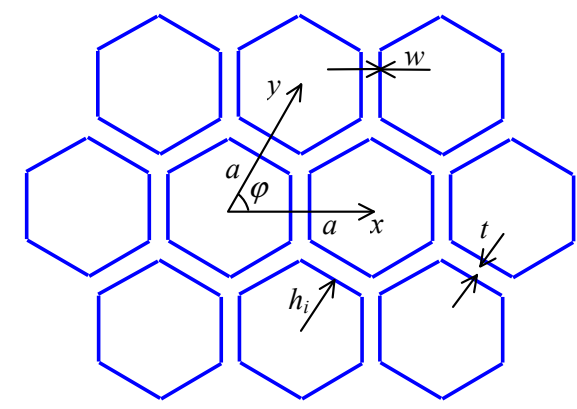

Figure 1. Hexagonal geometry of the considered frequency-selective surface (FSS). 
It can be seen that there is a periodic and symmetric pattern of perfectly electric conducting (PEC) metallic hexagons with $a$ representing periodicity on the $\mathrm{x}$ and $\mathrm{y}$ axes. Moreover, $t$ is the distance between metallic hexagons, $w$ is the width, and $h_{i}$ is the height. The FSS dimensions were selected using a parameter study that led to these optimal values: $w=0.4 \mathrm{~mm}, t=1.3 \mathrm{~mm}$, and $a=11.1 \mathrm{~mm}$.

\subsubsection{Wallpaper with Gypsum Wall}

In this case, for evaluation of the wallpaper's performance, the simulations employed a structure that consisted of FSS attached to a gypsum wall, as shown in Figure 2. The assumed thickness of the wall was $T=51.5 \mathrm{~mm}$ with a relative conductivity of $\sigma=0.0084 \mathrm{Am}^{-2}$ and a permittivity of $\varepsilon_{r}=5$.

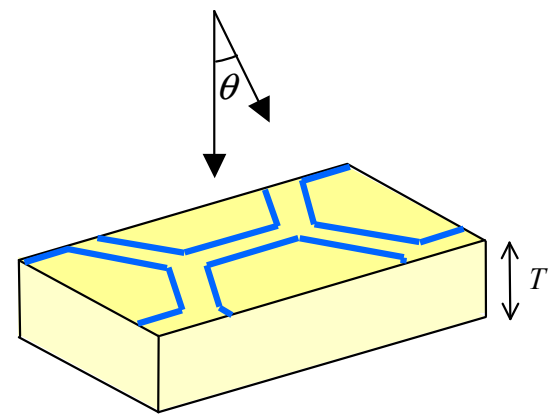

Figure 2. Scheme of the wallpaper attached to the gypsum wall.

This F-S wall's transmission and reflection coefficients, as expressed in [14], are presented in Figures 3 and 4, respectively, in $\mathrm{dB}$, being a function of frequency for a variety of incidence angles, as well as for both soft (horizontal) and hard (vertical) polarizations. It should be noted that, in this work, as indicated in [14], the so-called soft polarization is considered when the electric field is perpendicular to the plane of incidence (defined as the plane formed by a unit vector normal to the reflecting interface and the vector in the direction of incidence). This way, since the electric field is parallel to the interface, it is also known as horizontal. On the other hand, the so-called hard polarization is considered when the electric field is parallel to the plane of incidence. Since, in this case, a component of the electric field is perpendicular to the interface when the magnetic field is parallel to the interface, it is also known as vertical. The incidence angles shown in the plots $(\theta)$ correlate with the broadside (BS) direction shown in Figure 2. These plots also illustrate, for comparison purposes, an instance where BS incidence is assumed for the gypsum wall alone without wallpaper.

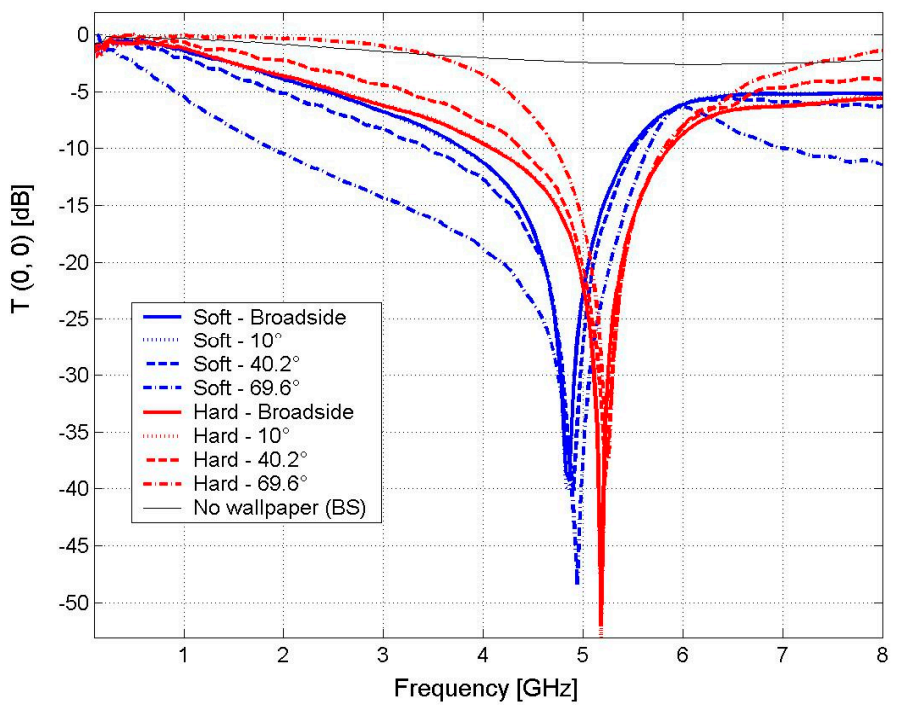

Figure 3. Transmission coefficient for the structure considered in Figure 2. 


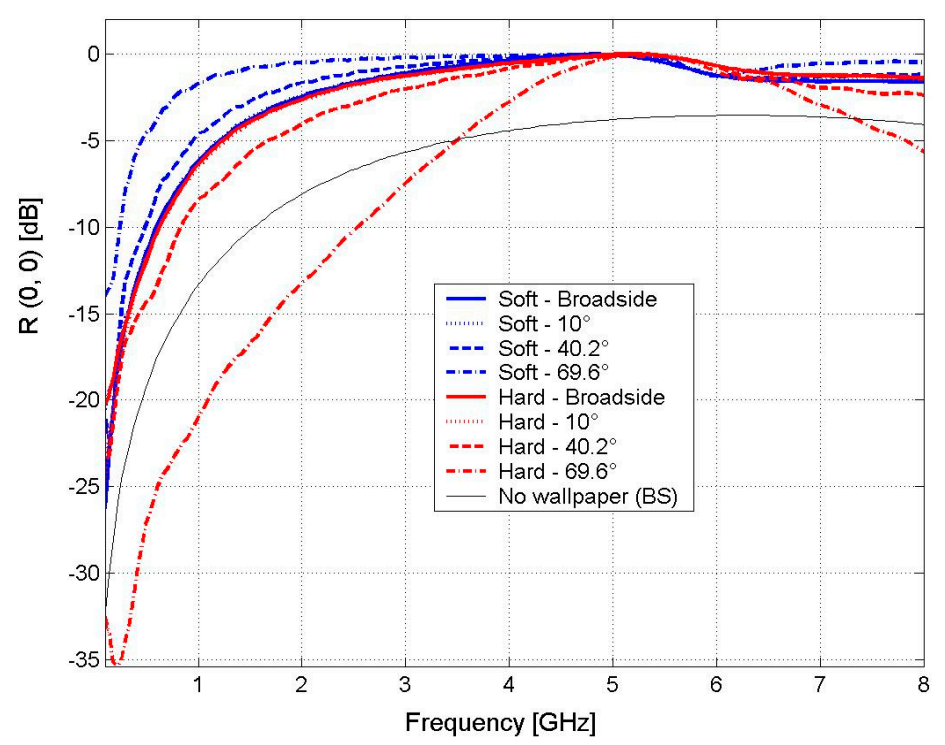

Figure 4. Reflection coefficient for the structure considered in Figure 2.

It may be seen that at $5 \mathrm{GHz}$ an extra transmission attenuation of $20 \mathrm{~dB}$ appears for both polarizations in comparison to the wall without wallpaper, as intended. Moreover, with angles of incidence ranging from BS to $69.6^{\circ}$, resonant frequencies vary only by $0.4 \mathrm{GHz}$. On the other hand, an almost perfect reflection is observed at $5 \mathrm{GHz}$ when considering the F-S wall.

The transmission and reflection coefficients of the gypsum wall with the wallpaper are given in Figures 5 and 6, respectively, as a function of the angle of incidence $\theta$ specified in Figure 2, assuming a frequency of $5 \mathrm{GHz}$, for both soft and hard polarizations. The curves were obtained by interpolating 12 different values corresponding to 12 different angles of incidence-equally spaced along the range from $0^{\circ}$ to $80^{\circ}$-through cubic spline data interpolation performed with MATLAB. It can be noted how the good transmission and reflection characteristics for the mentioned frequency remain valid for the entire range of angles considered.

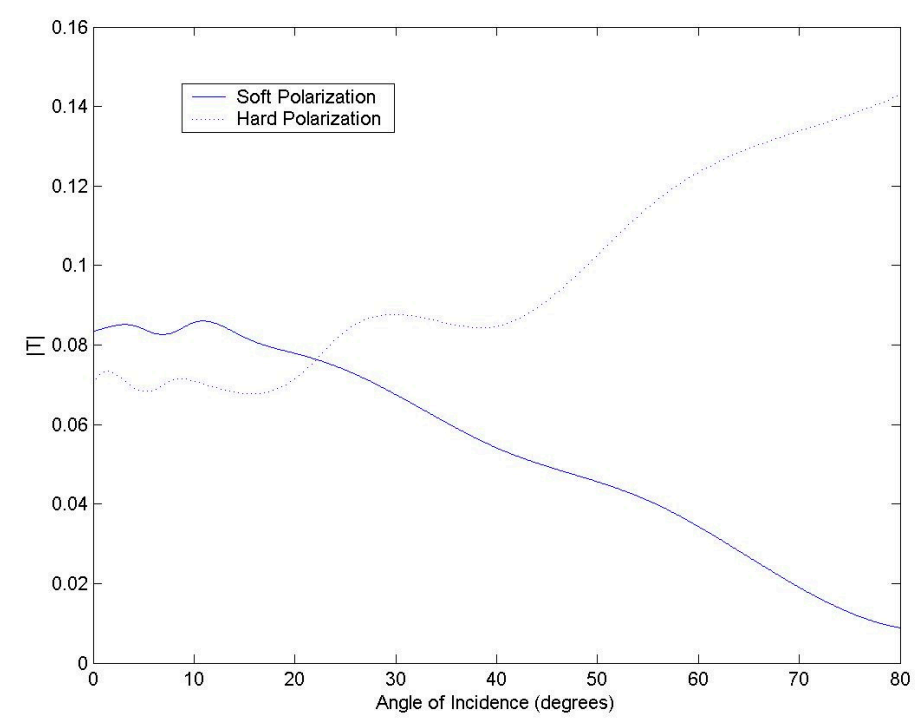

Figure 5. Transmission coefficient for the structure considered in Figure 2 for frequency $=5 \mathrm{GHz}$. 


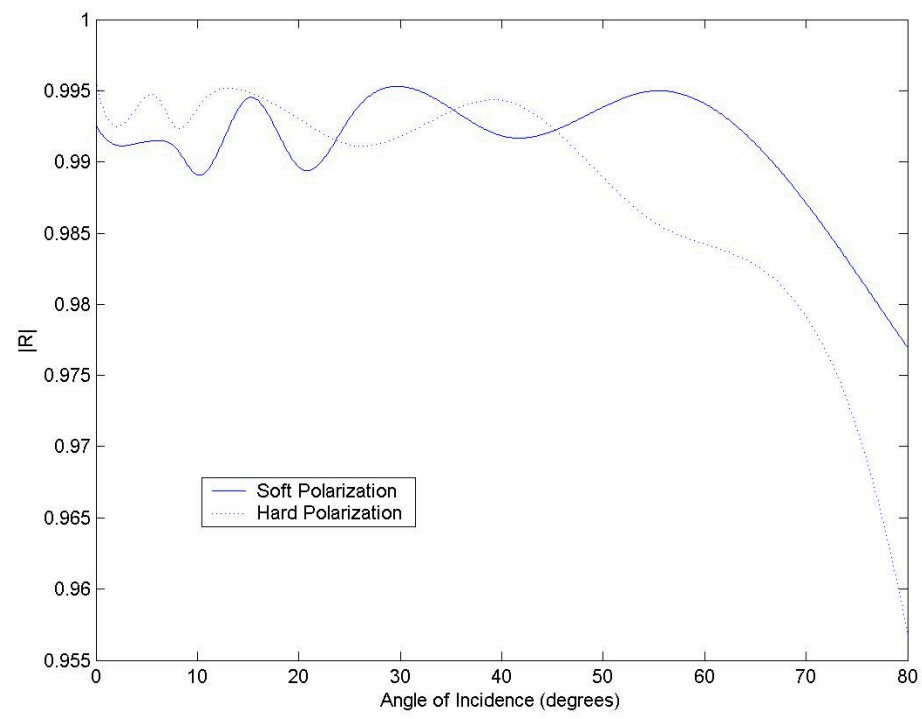

Figure 6. Reflection coefficient for the structure considered in Figure 2 for frequency $=5 \mathrm{GHz}$.

\subsubsection{Gypsum Wall Only}

For comparison purposes, a similar analysis to that undertaken in the previous subsection for the F-S wall is next performed considering the gypsum wall only (again, $\mathrm{T}=51.5 \mathrm{~mm}, \varepsilon \mathrm{r}=5$, and $\left.\sigma=0.0084 \mathrm{Am}^{-2}\right)$, as indicated in Figure 7.

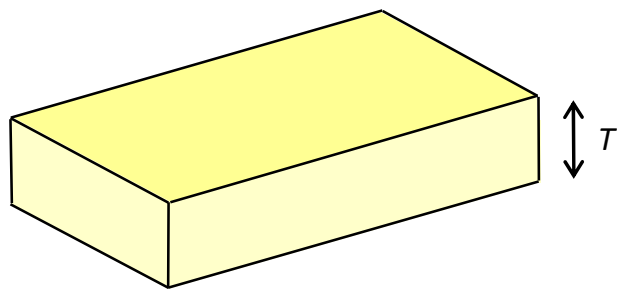

Figure 7. Scheme of the considered gypsum wall.

Therefore, the corresponding transmission and reflection coefficients are depicted in Figures 8 and 9, respectively, as a function of frequency, for different angles of incidence, and both soft and hard polarizations.

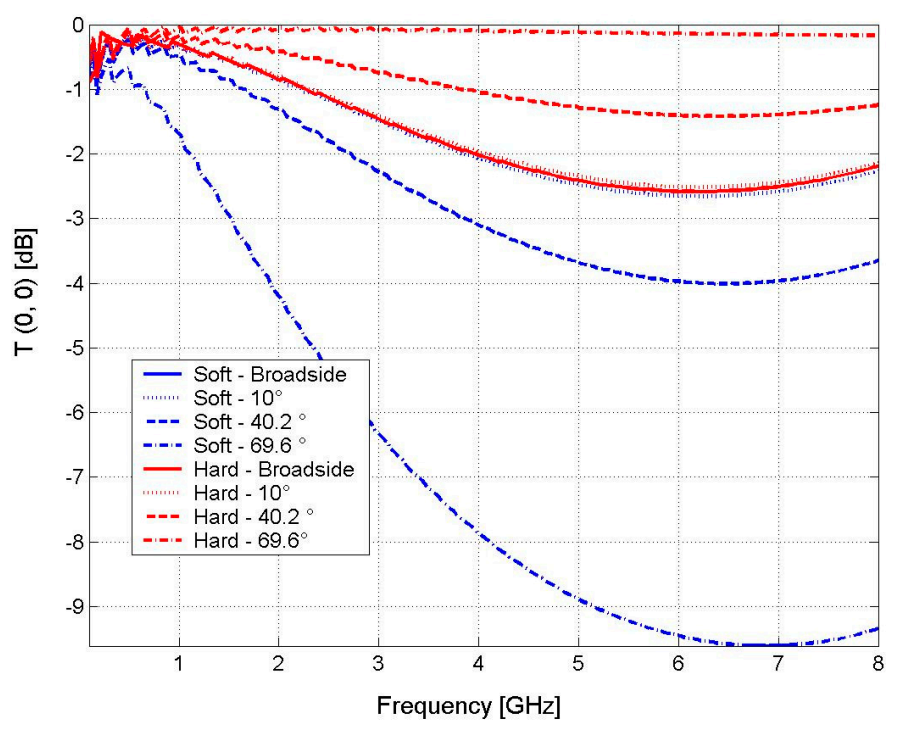

Figure 8. Transmission coefficient for the structure considered in Figure 7. 


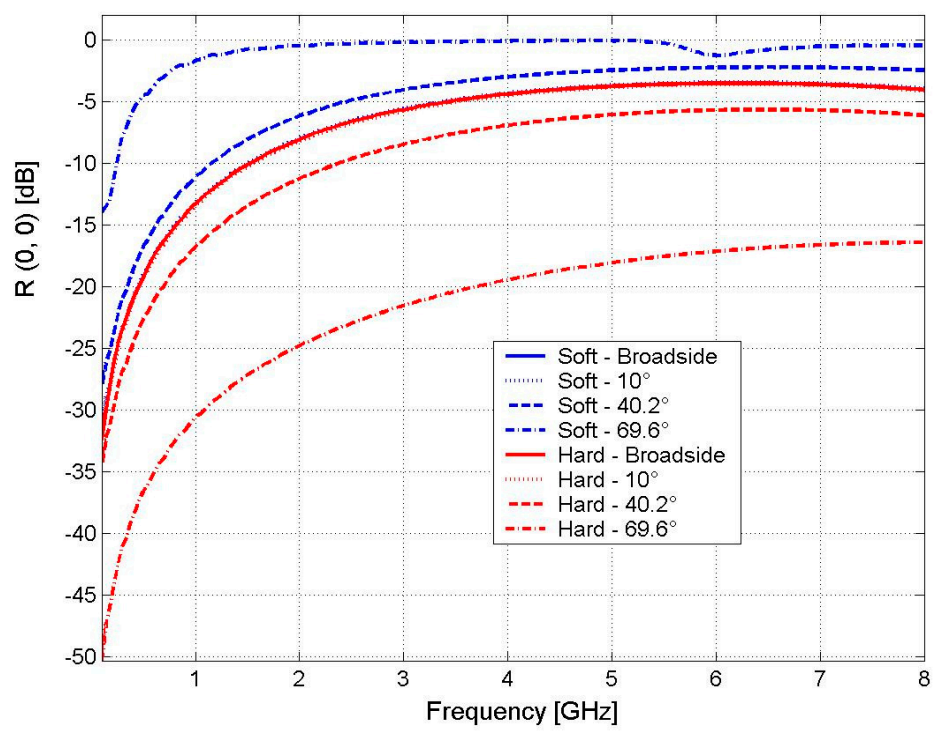

Figure 9. Reflection coefficient for the structure considered in Figure 7.

In addition, the transmission and reflection coefficients are depicted as a function of the angle of incidence, considering a frequency of $5 \mathrm{GHz}$, in Figures 10 and 11, respectively, for both soft and hard polarizations.

It can be noted from Figures $8-11$ that the transmission through a common gypsum wall is high at $5 \mathrm{GHz}$ compared to the wallpaper case and, moreover, the attenuation in signal strength significantly varies when different angles of incidence are considered.

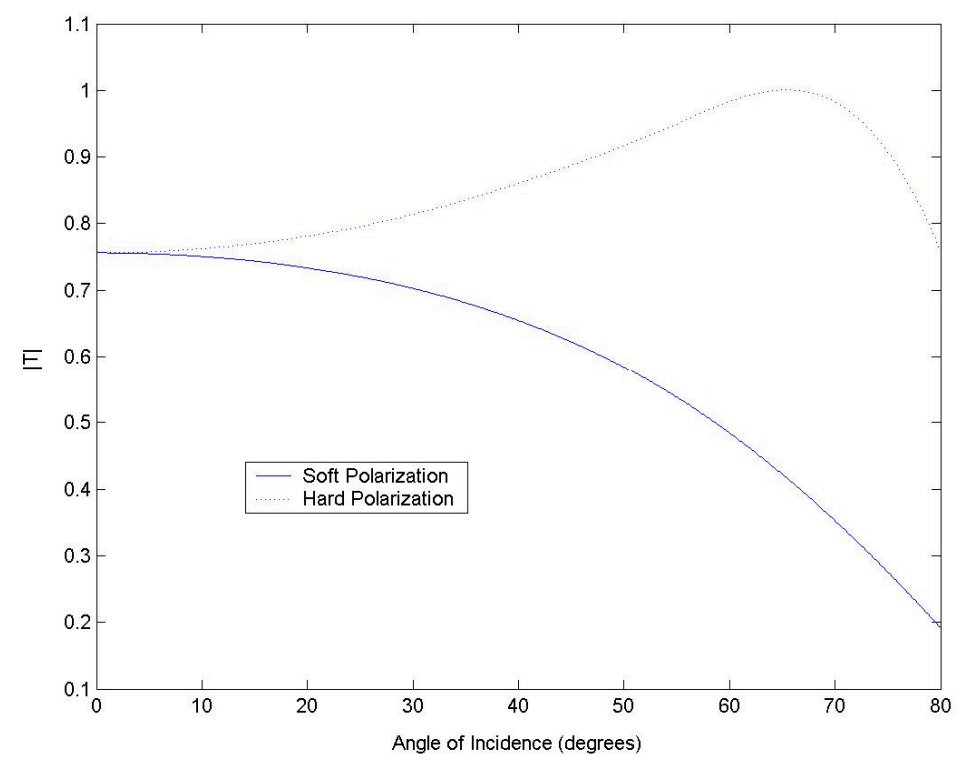

Figure 10. Transmission coefficient for the structure considered in Figure 7 for frequency $=5 \mathrm{GHz}$. 


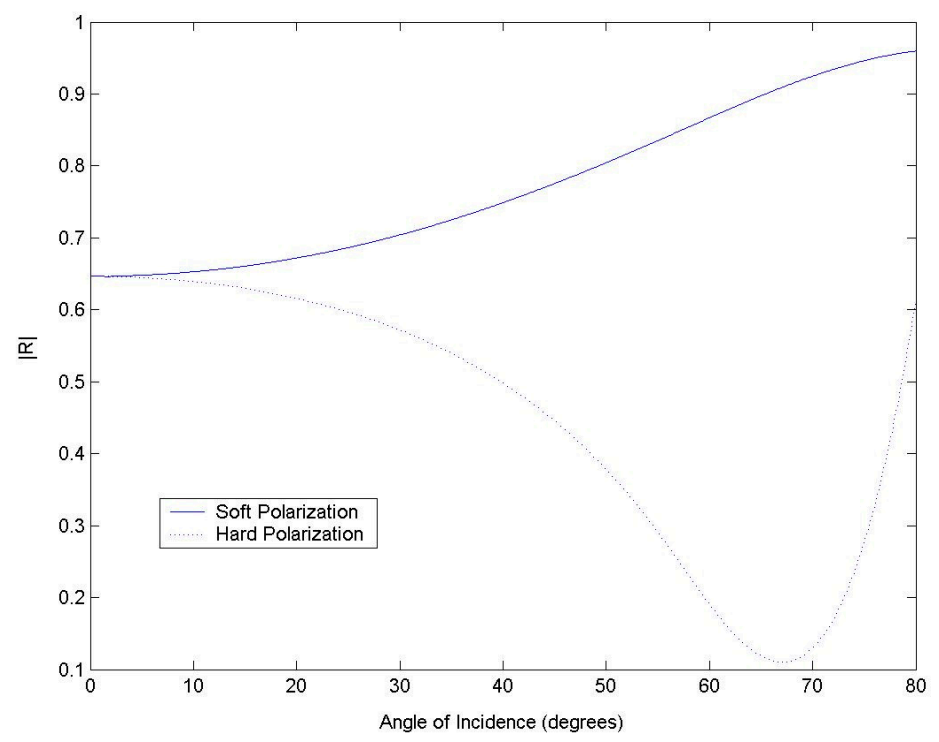

Figure 11. Reflection coefficient for the structure considered in Figure 7 for frequency $=5 \mathrm{GHz}$.

\subsubsection{Wallpaper Attached onto Other Structures}

\section{Wallpaper with Gypsum-Wood Wall}

In order to check the versatility of the designed wallpaper, simulations were performed considering that the latter is covering a gypsum-wood wall, as can be seen in Figure 12. Parameters of $\varepsilon_{r}=5$, $\sigma=0 \mathrm{Am}^{-2}$, and a thickness of $T^{\prime}=102.9 \mathrm{~mm}$ were assumed for the wood layer. Dimensions and material properties for the gypsum layer remained the same as those considered in previous sections.

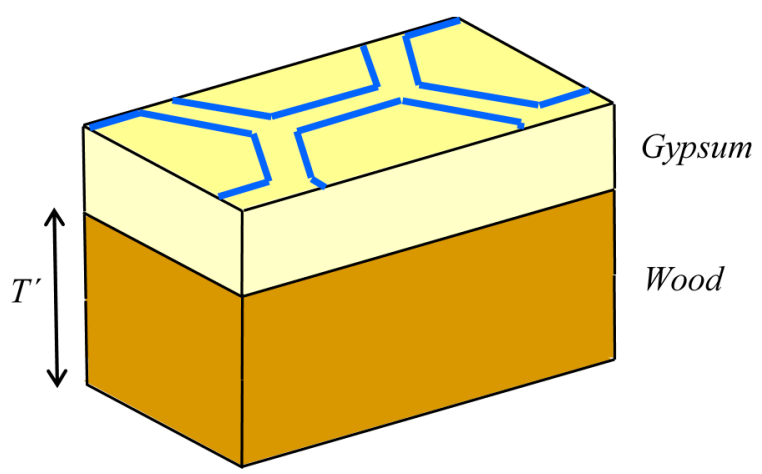

Figure 12. Scheme of the wallpaper attached to a gypsum-wood wall.

The transmission and reflection coefficients for the described structure can be observed in Figures 13 and 14, respectively, as a function of frequency, considering BS incidence.

It should be noted how the resonant frequencies are not shifted when considering the wallpaper with a gypsum-wood structure and, furthermore, their difference in attenuation as compared to the wallpaper-gypsum wall case is only $5 \mathrm{~dB}$ for soft polarization, therefore confirming that the performance of the designed wallpaper remains efficient with a gypsum-wood structure. Specifically, a transmission loss of around $45 \mathrm{~dB}$ can be found for both polarizations for the gypsum-wood structure around $5 \mathrm{GHz}$. Furthermore, the $-18 \mathrm{~dB}$ bandwidth at resonant frequencies is approximately $350 \mathrm{MHz}$, which should be sufficient for band filtering purposes. On the other hand, the desired perfect reflection at $5 \mathrm{GHz}$, which was achieved with the gypsum wall, is maintained at $5 \mathrm{GHz}$ when considering a gypsum-wood structure. 


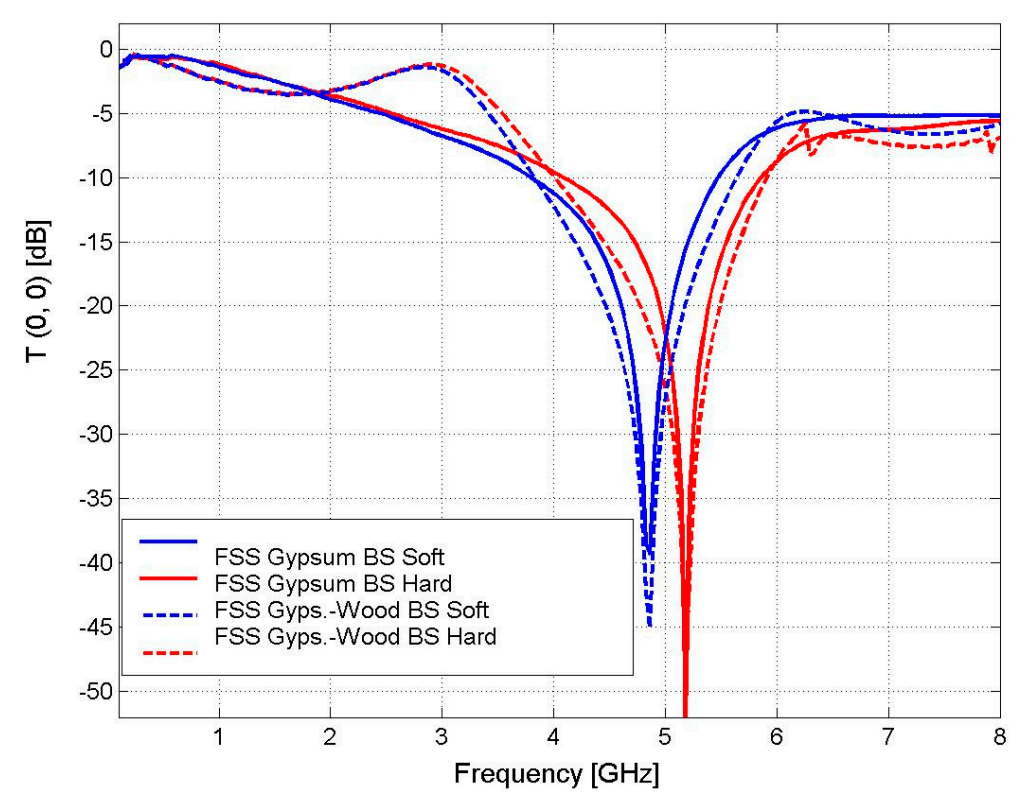

Figure 13. Transmission coefficient for the structure considered in Figure 12.

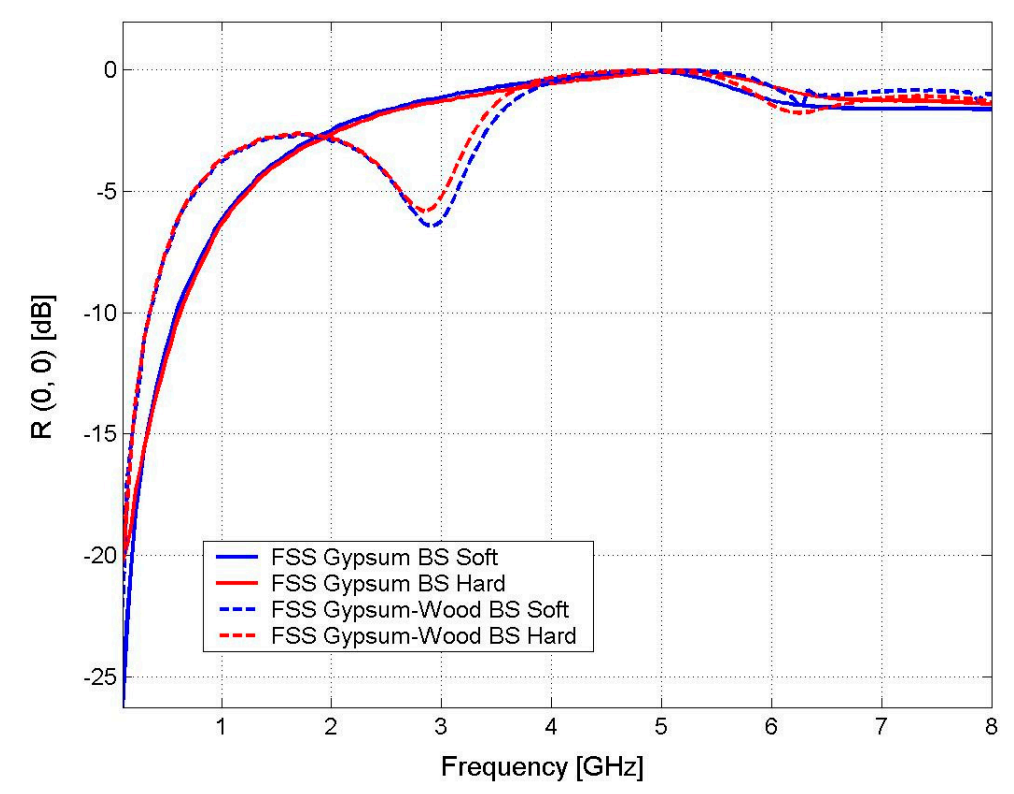

Figure 14. Reflection coefficient for the structure considered in Figure 12.

Wallpaper with Gypsum-Air-Gypsum Wall

The same analysis described in the previous subsection was carried out considering the wallpaper attached to a gypsum-air-gypsum wall, as shown in Figure 15.

Dimensions and material properties for the gypsum layers remained the same as those considered in previous sections and a separation of $51.5 \mathrm{~mm}$ (air) was assumed between both boards.

Again, it can be seen in Figures 16 and 17 how the band-stop response of the newly considered structure is still exhibiting the good properties seen in the wallpaper-gypsum wall case regarding the blocking/confining of the signal. Specifically, a transmission loss of around $37 \mathrm{~dB}$ and $47 \mathrm{~dB}$ for soft and hard polarizations, respectively, can be observed for the gypsum-wood structure around $5 \mathrm{GHz}$. Moreover, the $-18 \mathrm{~dB}$ bandwidth at resonant frequencies is approximately $400 \mathrm{MHz}$, which, again, should be sufficient for band filtering purposes. On the other hand, as observed in the gypsum-wood case, the desired perfect reflection at $5 \mathrm{GHz}$, which was achieved with the gypsum wall, is again kept at $5 \mathrm{GHz}$ when considering a gypsum-air-gypsum structure. 


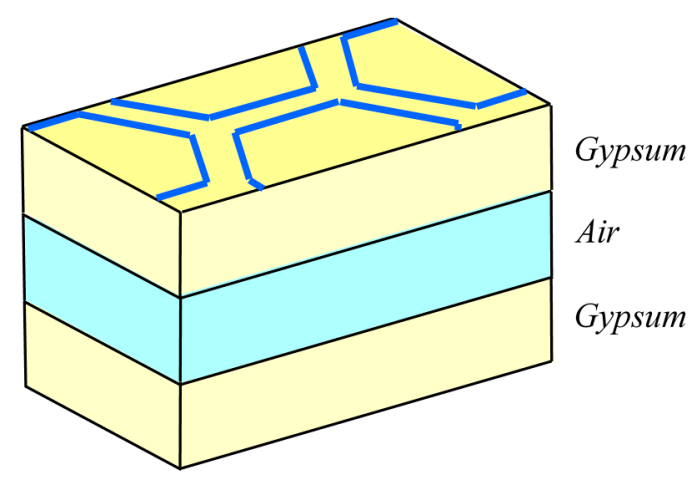

Figure 15. Scheme of the wallpaper attached to a gypsum-air-gypsum wall.

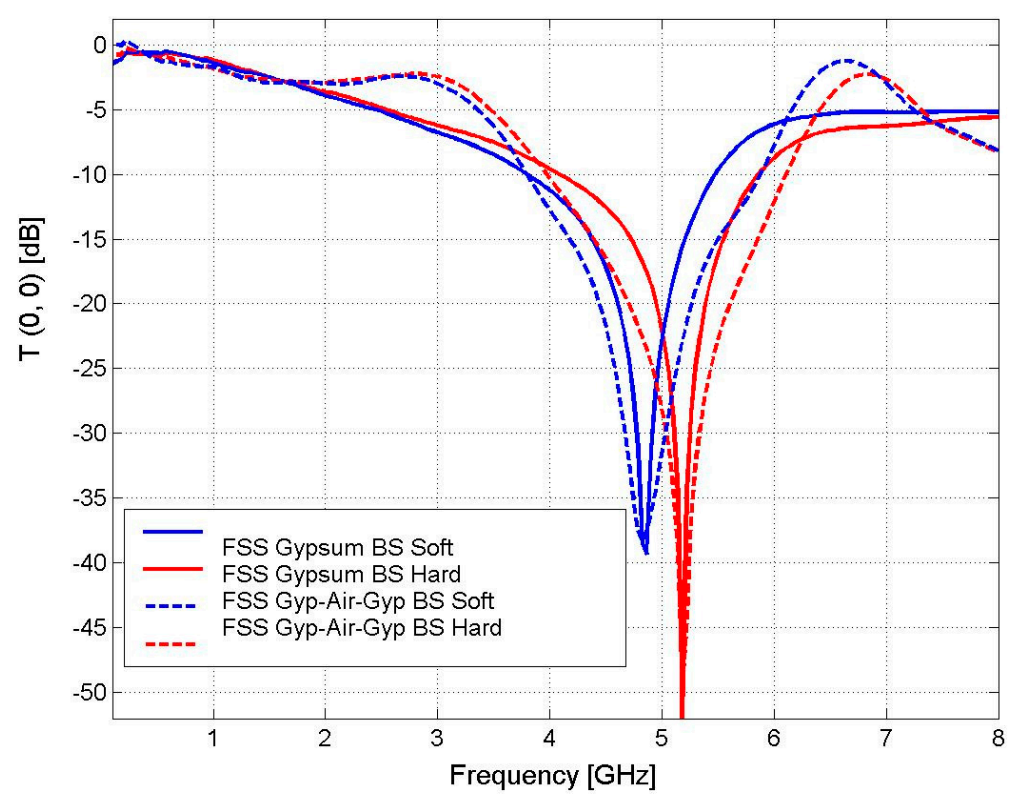

Figure 16. Transmission coefficient for the structure considered in Figure 15.

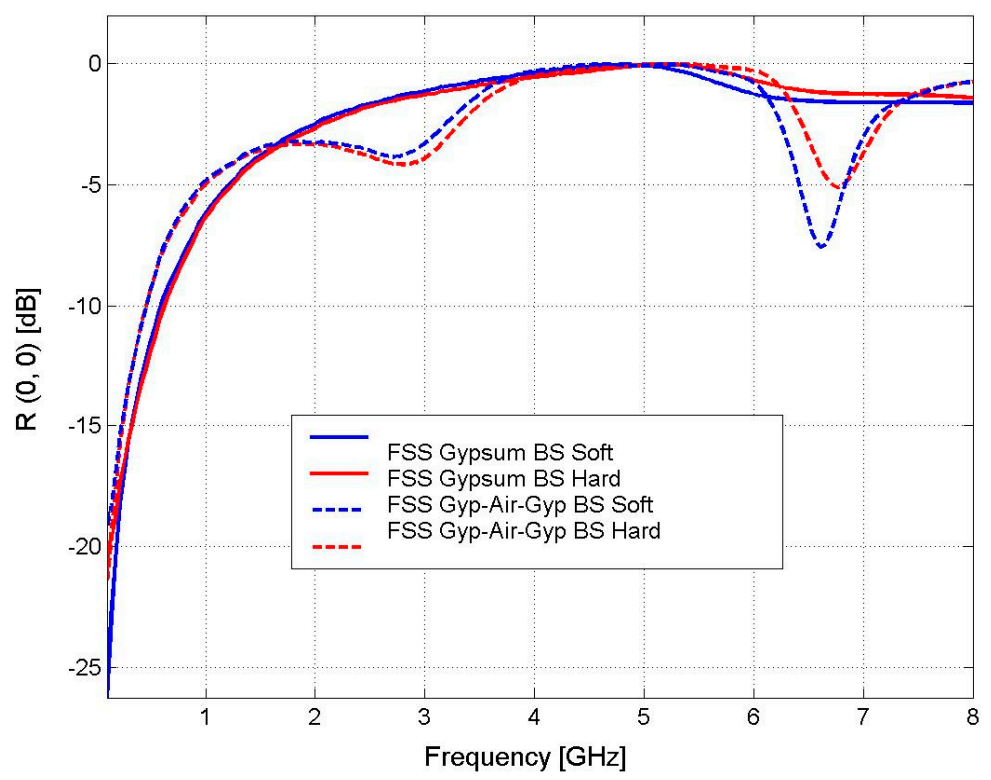

Figure 17. Reflection coefficient for the structure considered in Figure 15. 


\section{Ray-Launching Method}

To achieve the results shown in this paper, the simulations were performed employing a ray-launching program centered on ray optics and the uniform theory of diffraction (UTD). With ray-launching techniques, rays are shot at various discrete angles that undergo interaction with any objects in situ during propagation. Thus, the rays that have been launched undergo reflection, transmission, diffraction, and scattering across their environment. Random rays passing near the location of the receiver are employed for predicting the true propagation pulse and thus we may evaluate the total field registered by the receiver as being a coherent summation of every contribution that impinges upon it.

The ray-launching tool employed in this research may be used for analyzing both MIMO and SISO systems. With the MIMO system results, the assumption is made that there is an equal number of receiving antennae $(\mathrm{Nr})$ and transmitting antennae $(\mathrm{Nt})$, meaning $\mathrm{N}=\mathrm{Nt}=\mathrm{Nr} . \mathrm{N} \times \mathrm{N}$ simulations were performed for every receiver position for obtaining the channel transfer matrix $\left(G_{N \times N}\right)$. For each simulation, a calculation is made of the coefficient $G(n, m)$, being the coherent sum of every ray received by the antenna $m$ with transmissions only coming from the antenna $n$.

The received rays taken into consideration comprise: direct $\left(E_{1}\right)$, multiple reflected $\left(E_{2}\right)$, multiple transmitted $\left(E_{3}\right)$, multiple diffracted $\left(E_{4}\right)$, multiple reflected/diffracted $\left(E_{5}\right)$, multiple transmitted/ diffracted $\left(E_{6}\right)$, and multiple transmitted/reflected $\left(E_{7}\right)$ rays. Thus, the matrix $\mathrm{G}$ for any specific receiver position is found with [15]:

$$
G(n, m)=\sum_{c=1}^{7} E_{c}(n, m)
$$

with direct, reflected, transmitted, diffracted, reflected/diffracted, transmitted/diffracted, and transmitted/ reflected components being ascertained with [15]:

$$
\begin{gathered}
E_{1}(n, m)=\frac{E_{o}}{r} e^{-j k r} \\
E_{2}(n, m)=\sum_{i=1}^{p} R_{i} \frac{E_{o}}{r_{i}} e^{-j k r_{i}} \\
E_{3}(n, m)=\sum_{i=1}^{p} T_{i} \frac{E_{o}}{r_{i}} e^{-j k r_{i}} \\
E_{4}(n, m)=\sum_{i=1}^{p} \frac{E_{o}}{s_{i}^{\prime}} e^{-j k s_{i}^{\prime}} D_{i} \sqrt{\frac{s_{i}^{\prime}}{s_{i}\left(s_{i}+s_{i}^{\prime}\right)}} e^{-j k s_{i}} \\
E_{5}(n, m)=\sum_{i=1}^{p} \frac{E_{o}}{s_{i}^{\prime}} e^{-j k s_{i}^{\prime}} D_{i} R_{i} \sqrt{\frac{s_{i}^{\prime}}{s_{i}\left(s_{i}+s_{i}^{\prime}\right)}} e^{-j k s_{i}} \\
E_{6}(n, m)=\sum_{i=1}^{p} \frac{E_{o}^{\prime}}{s_{i}^{\prime}} e^{-j k s_{i}^{\prime}} D_{i} T_{i} \sqrt{\frac{s_{i}^{\prime}}{s_{i}\left(s_{i}+s_{i}^{\prime}\right)}} e^{-j k s_{i}} \\
E_{7}(n, m)=\sum_{i=1}^{p} T_{i} R_{i} \frac{E_{o}}{r_{i}} e^{-j k r_{i}}
\end{gathered}
$$

where $E_{o}$ represents emitted field strength, $k$ the wave number, $p$ the total number of contributions considered, $r$ and $r_{i}$ the propagation path lengths from source $n$ to receiver $m, s^{\prime}$ the path length from source to the diffracting wedge, $s$ the path length from diffracting wedge to receiver, $D_{i}$ the diffraction coefficient for finitely conducting wedges shown in [16], $T_{i}$ the transmission coefficient, and $R_{i}$ the reflection coefficient. For this instance, $T_{i}$ and $R_{i}$ are dependent on the incident wave polarization, angle of incidence, conductivity, and permittivity.

For analytical purposes, the assumption is made that power is distributed equally for every transmitter and that the transmitter has no prior channel knowledge [17]. 


\section{Results and Discussion}

\subsection{SISO Case}

The deployment of the F-S wallpaper in an indoor scenario was undertaken employing the aforementioned ray-launching software; the first experiment was undertaken using an SISO system in a two-room environment, the top view of which is shown in Figure 18 (distances in meters).

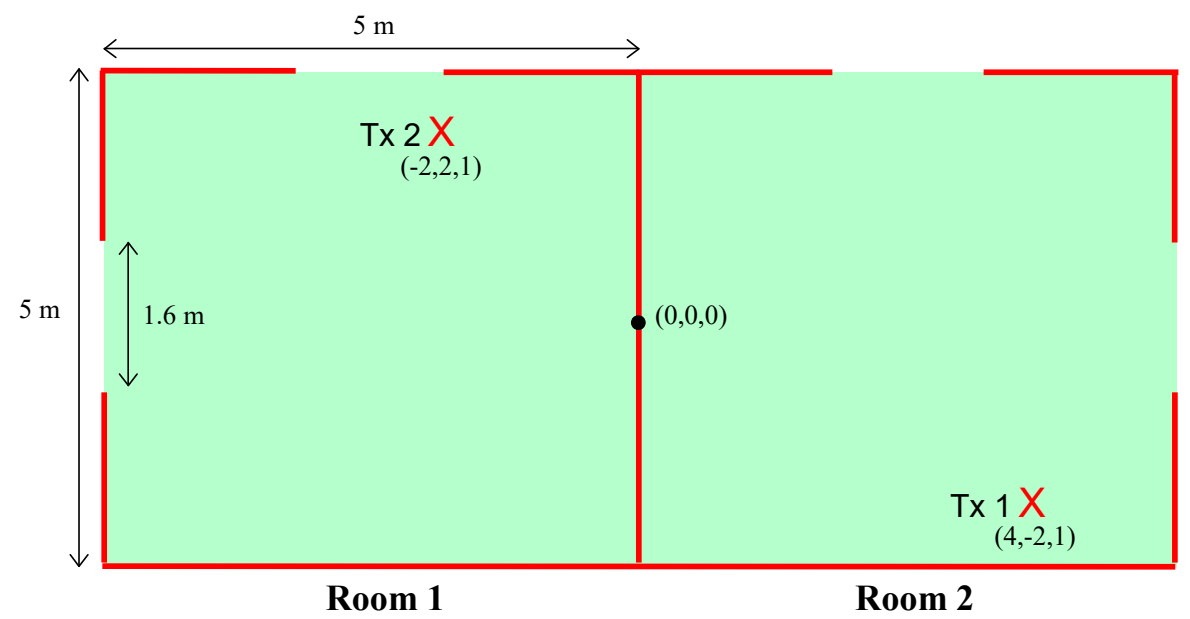

Figure 18. Geometry of rooms and transmitter locations (top view).

As may be observed, the floor comprises two $25 \mathrm{~m}^{2}$ rooms, each having two apertures corresponding to squared windows of $1.6 \mathrm{~m}$ width; the experiment assumes that the rooms have a height of $3 \mathrm{~m}$. The $\mathrm{T} \times 1$ transmitter is located within Room 2, providing coverage in that location. $\mathrm{T} \times 2$ is located within Room 1 so that when its signal enters Room 2, it becomes an interference.

As an example, in Figure 19, the signal-to-interference ratio (SIR), in dB, over every point at a horizontal plane of $1 \mathrm{~m}$ height in Room 2 is depicted considering the two cases in which (a) regular gypsum walls are considered and (b) wallpaper-gypsum walls are assumed. The following simulation parameters were taken into account in the ray-launching code:

- Omnidirectional antennas

- Frequency: $5 \mathrm{GHz}$

- Soft Polarization

- $\mathrm{T} \times 1$ and $\mathrm{T} \times 2$ bearing a $1 \mathrm{~m}$ height

- Number of rays launched: 7200

- Number of considered reflections: 14

- Number of considered reflections of diffracted rays: 4

- Number of considered transmissions: 4

- Number of considered transmissions of diffracted rays: 2.

Figure 20 shows the cumulative distribution function (CDF) for the signal-to-interference ratio (SIR) for every point of Room 2 for both instances of uncovered walls and walls covered with the designed wallpaper. An assumption is made that the antennae are omnidirectional, the frequency is $5 \mathrm{GHz}$, both transmitters are $1 \mathrm{~m}$ in height (the third coordinator that may be observed in the positioning of the transmitters in Figure 18), and that the polarization is soft (horizontal). 


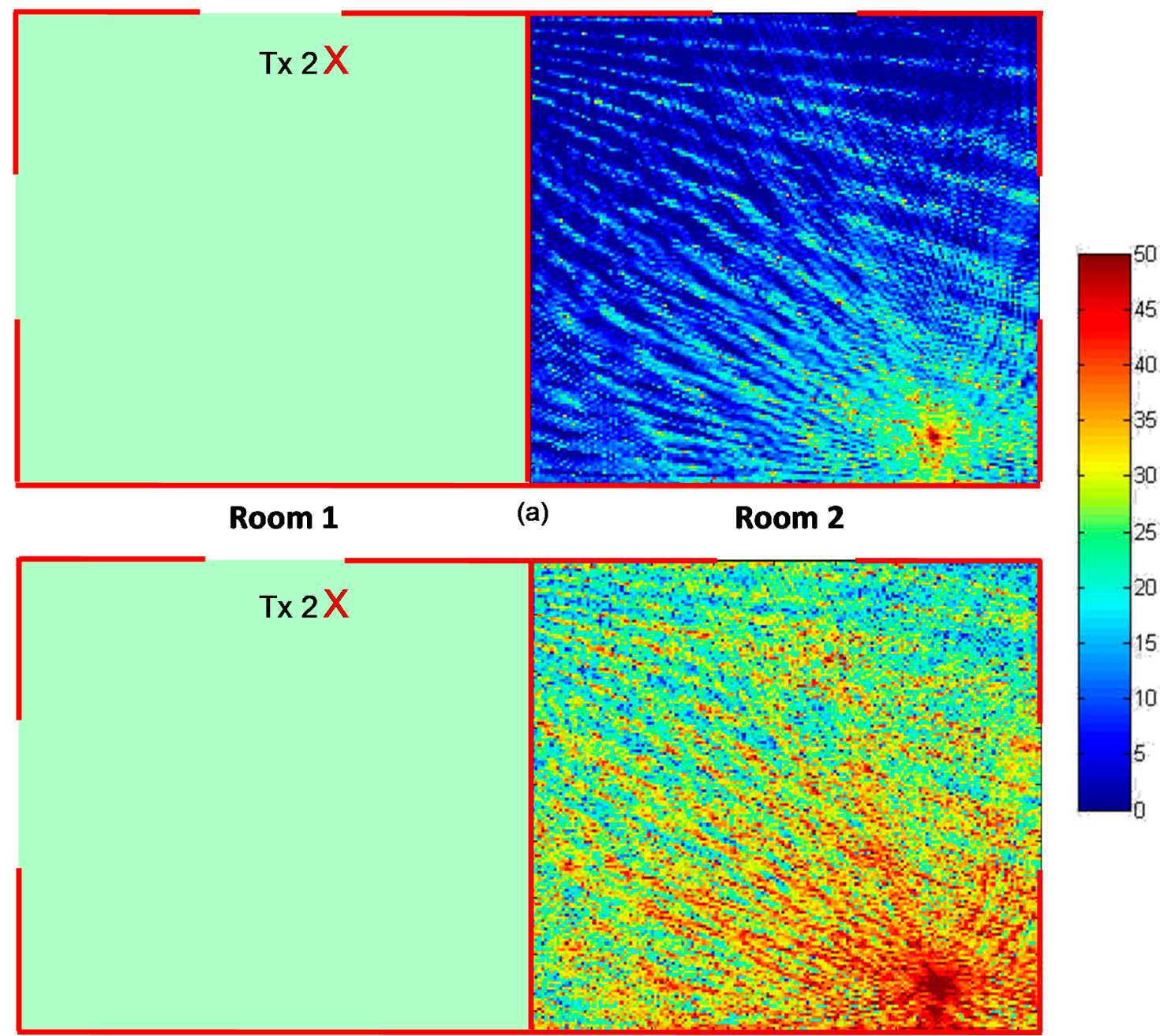

\section{Room 1}

(b)

Room 2

Figure 19. The signal-to-interference ratio (SIR) over Room 2 (dB) considering (a) regular gypsum walls (b) F-S walls.

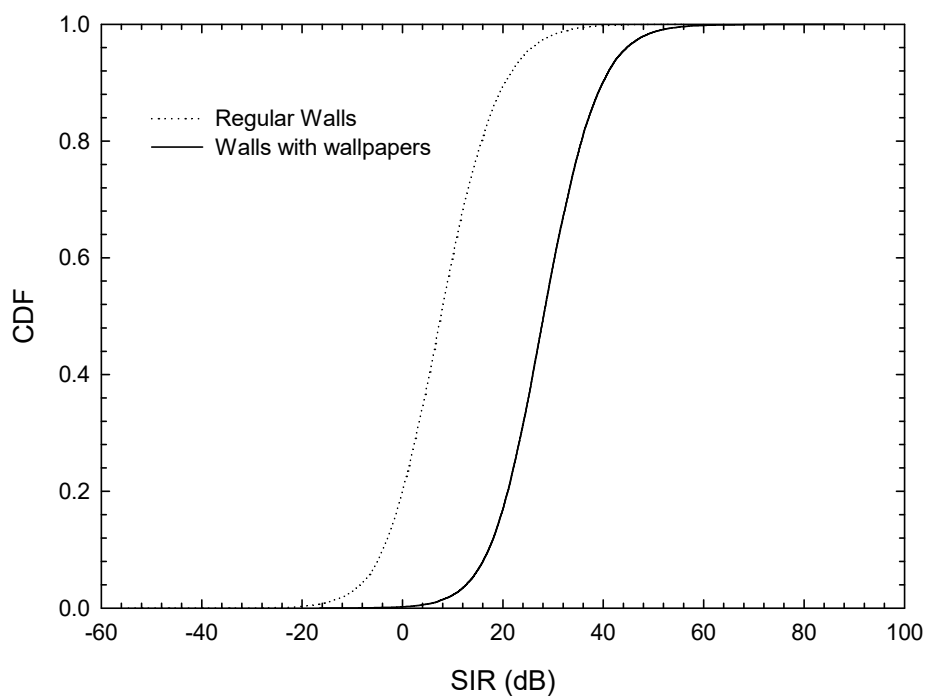

Figure 20. Cumulative distribution function (CDF) of the SIR in Room 2. 
We can see that the F-S walls create an improvement of approximately $20 \mathrm{~dB}$ in the SIR. This agrees with predicted values, as shown in Figure 3.

For evaluation of the power delay profile (PDP) from transmitter to receiver in Room 2 for both papered and unpapered walls, we considered the scheme illustrated in Figure 21, containing transmitter $\mathrm{T} \times 1$ and receiver $\mathrm{R} \times 1$.

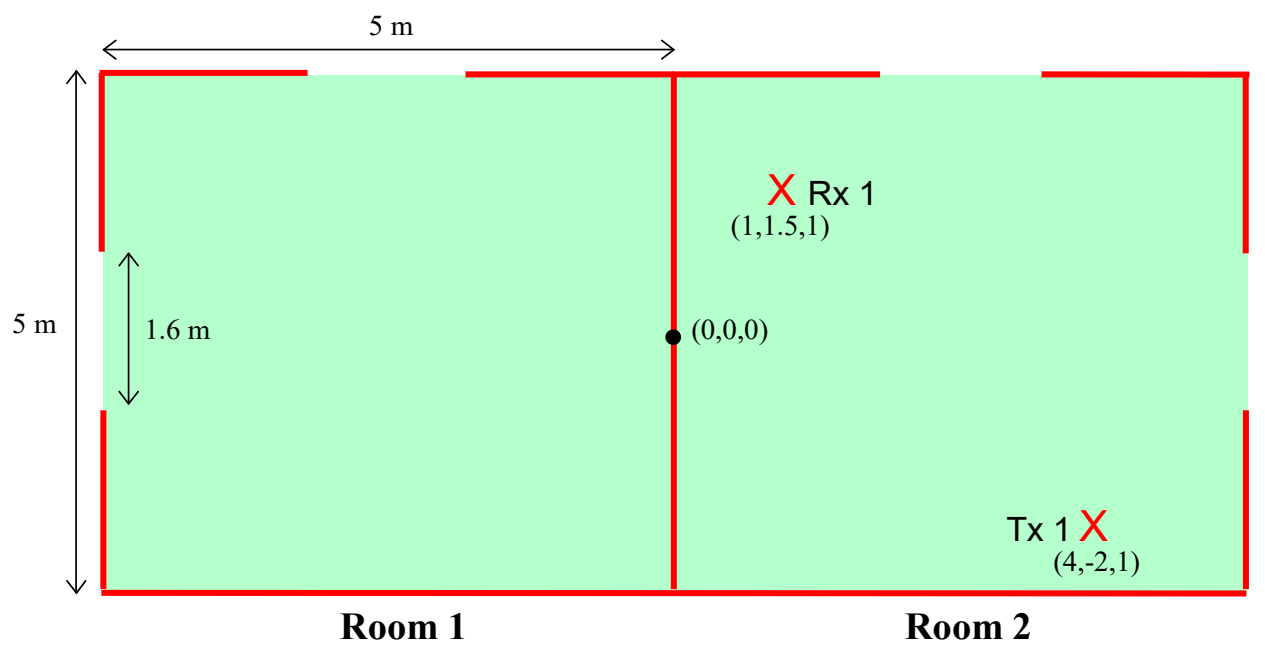

Figure 21. Geometry of rooms and transmitter/receiver locations (top view).

For this instance, the aforementioned PDP may be seen in Figure 22 for both papered and unpapered walls. The simulation parameters considered in the ray-launching code were the following:

- Omnidirectional antennas

- Frequency: $5 \mathrm{GHz}$

- Hard Polarization

- $\mathrm{T} \times 1$ and $\mathrm{T} \times 2$ bearing a $1 \mathrm{~m}$ height

- Number of rays launched: 7200

- Number of considered reflections: 14

- Number of considered reflections of diffracted rays: 4

- Number of considered transmissions: 4

- Number of considered transmissions of diffracted rays: 2 .

The curves show 20 realizations on average harvested at various frequencies between 4.9 and $5.1 \mathrm{GHz}$ measured in $0.01 \mathrm{GHz}$ increments. Additionally, low-pass filtering with $40 \mathrm{MHz}$ was undertaken within the frequency domain with a Blackman window for all realizations. An assumption is made that the antennae are omnidirectional, the frequency is $5 \mathrm{GHz}$, and both transmitters are $1 \mathrm{~m}$ in height. It may be seen that the PDP curve generated in the instance of the wall being papered with F-S wallpaper has a slower decrease when the time delay increases. This means that a wide range of outstanding paths come to the receiver, which increases the root-mean-square (r.m.s) delay spread. An r.m.s. delay spread of $22 \mathrm{~ns}$ can be ascertained from the papered wall results (coherence bandwidth approximately $7 \mathrm{MHz}$ ), in comparison to the unpapered wall, which has a value of $4 \mathrm{~ns}$ (coherence bandwidth of approximately $42 \mathrm{MHz})$. 


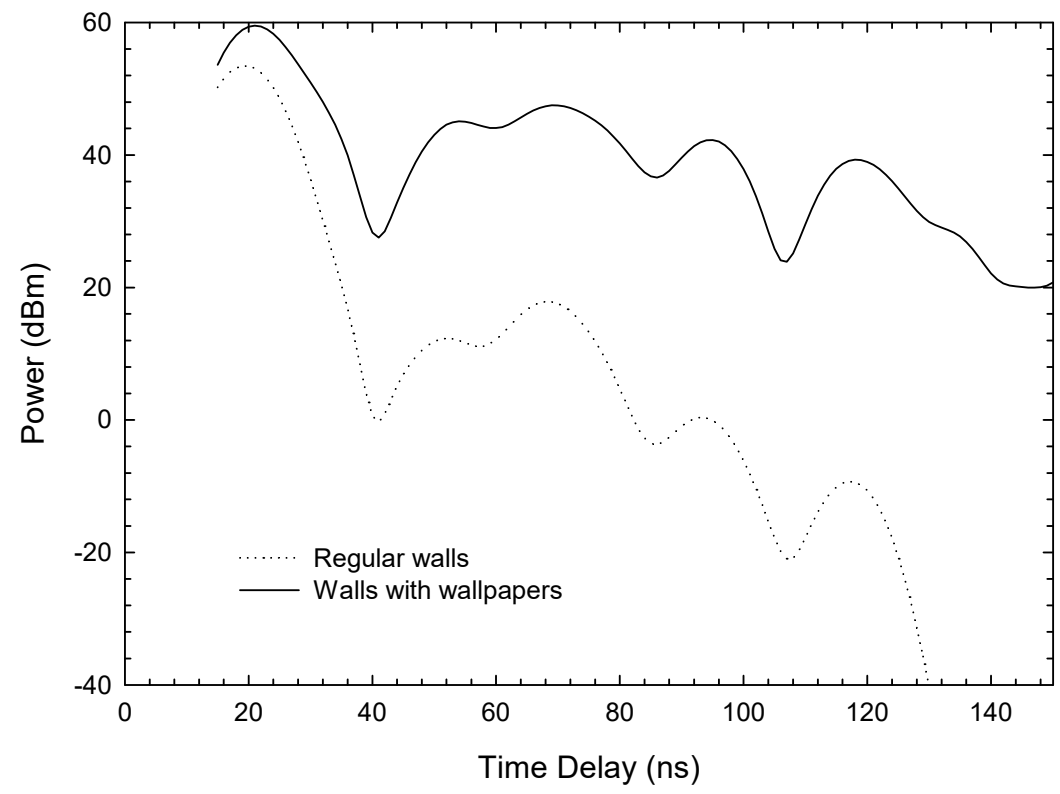

Figure 22. Power delay profile for the geometry in Figure 20.

\subsection{MIMO Case}

A further analysis of the employment of F-S wallpaper in an indoor scenario, but this time using a MIMO system, was undertaken. Figure 23 shows the layout in this instance, with Room 2 holding the transmitter and receiver $\mathrm{T} \times 1$ and $\mathrm{R} \times 1$, respectively, comprising a quartet of omnidirectional antennae spaced at $\lambda / 2$.

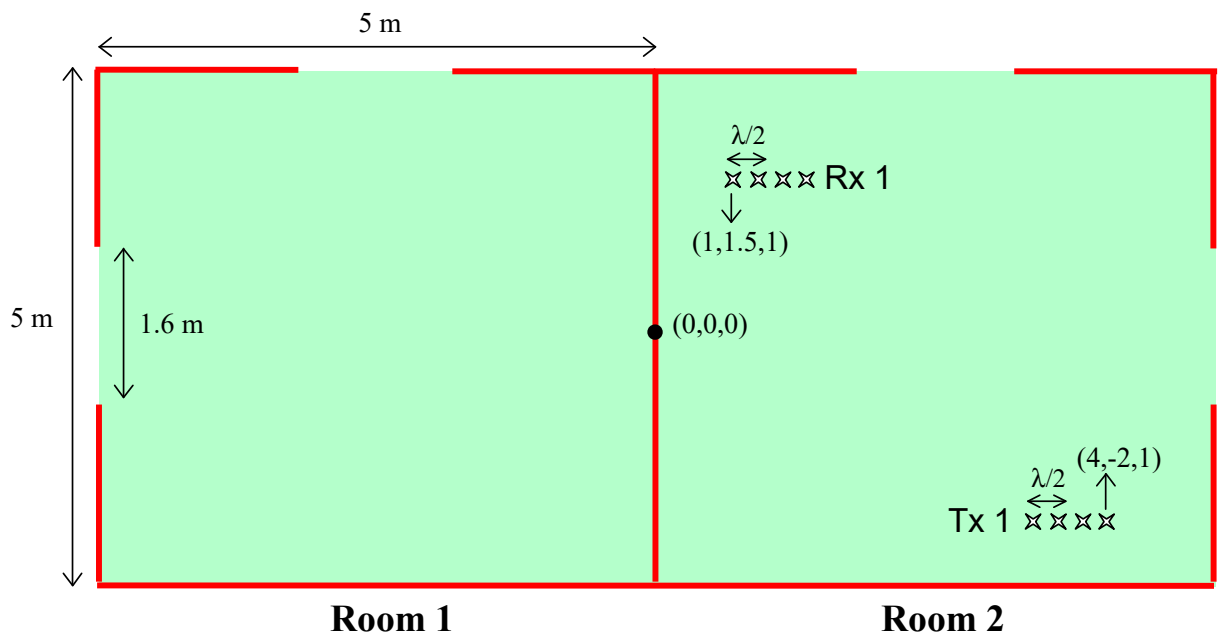

Figure 23. Geometry of two rooms and multiple-input multiple-output (MIMO) transmitter/receiver locations (top view).

Using this setup, Figure 24 shows the singular values (lambda 1 to 4 ) for the channel transfer matrix $G$ (square roots of the eigenvalues of $G^{T} G$ and $G G^{T}$ ) as a function of frequency with both unpapered and papered walls. The simulation parameters considered in this case in the ray-launching code were the following:

- Omnidirectional antennas

- Hard Polarization

- $4 \mathrm{Tx}$ and $4 \mathrm{Rx}$ bearing a $1 \mathrm{~m}$ height

- Number of rays launched: 7200 
- Number of considered reflections: 9

- Number of considered reflections of diffracted rays: 4

- Number of considered transmissions: 4

- Number of considered transmissions of diffracted rays: 3 .

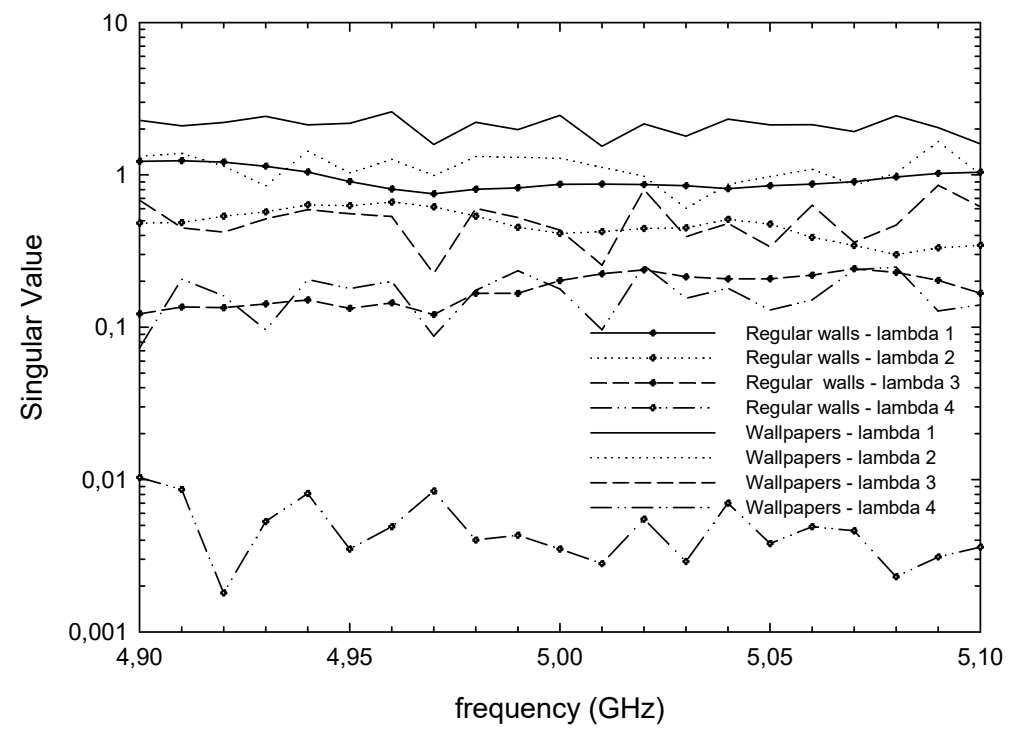

Figure 24. Singular values for the channel transfer matrix considering the geometry in Figure 23.

We can say that with unpapered walls there is a trio of main singular values (the fourth being negligible for practical purposes). When the F-S paper covers every wall, there is an increase in the magnitude of all four singular values, with the fourth value being emphasized, so that, in this instance, we have four relevant subchannels rather than three. In this instance, an extra degree of freedom is added in comparison to the unpapered walls, and thus the capacity of the radio channel is increased.

In order to further the previous study, the radiation diagrams calculated from the eigenvectors of $\mathrm{GG}^{\mathrm{T}}$ and $\mathrm{G}^{\mathrm{T}} \mathrm{G}$, concerning the antenna arrays $\mathrm{T} \times 1$ and $\mathrm{R} \times 1$, can be observed in Figure 25 for a frequency of $5 \mathrm{GHz}$ and the two types of walls under study.
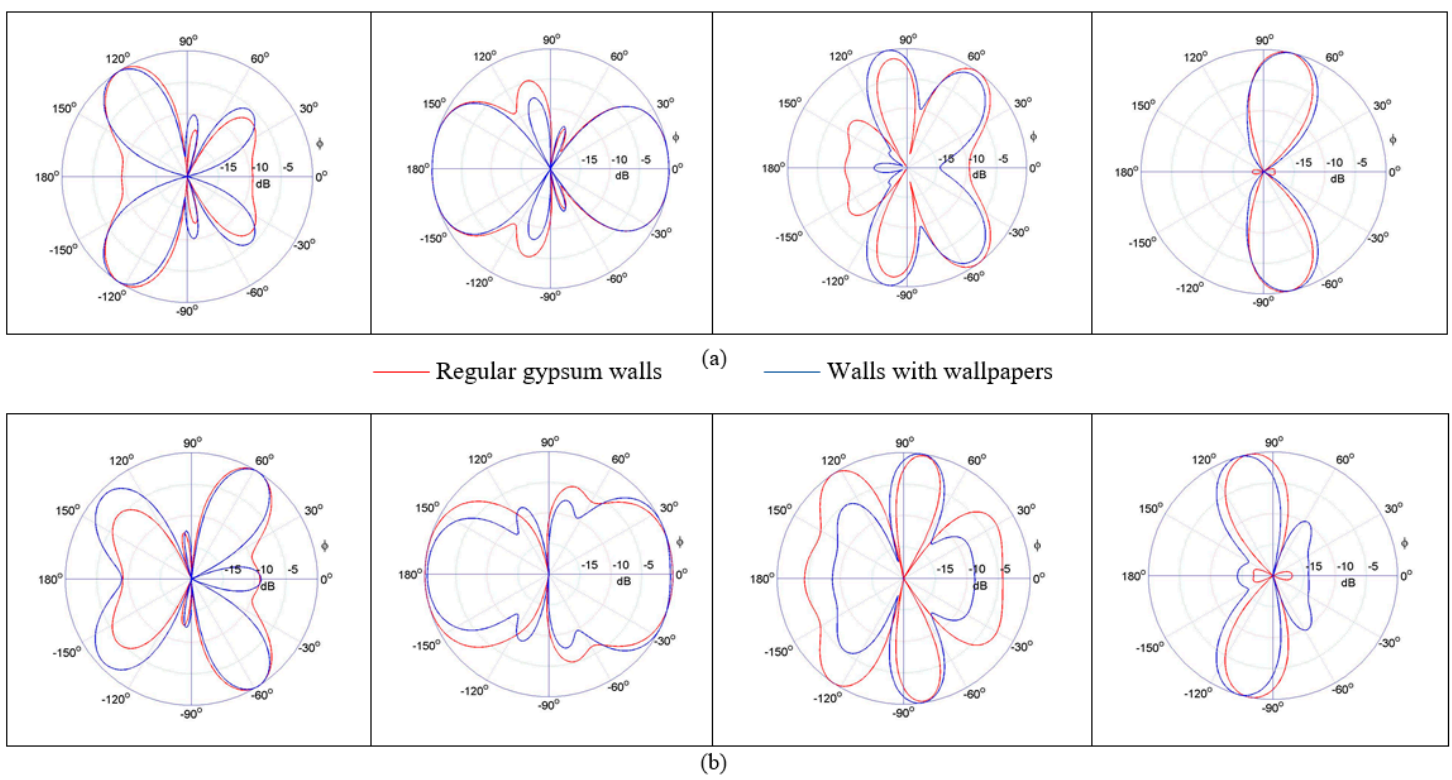

Figure 25. Radiation diagrams from the eigenvectors of $\mathrm{GG}^{\mathrm{T}}$ and $\mathrm{G}^{\mathrm{T}} \mathrm{G}$ applied to (a) the transmitter $\mathrm{T} \times 1$ and $(\mathbf{b})$ the receiver $\mathrm{R} \times 1$. Frequency $=5 \mathrm{GHz}$. 
As expected, the radiation diagrams located on the very left column-for both $\mathrm{T} \times 1$ and $\mathrm{R} \times$ 1 -which were calculated from the first eigenvectors of $\mathrm{GG}^{\mathrm{T}}$ and $\mathrm{G}^{\mathrm{T}} \mathrm{G}$, present the lobes (at $120^{\circ}$ in the transmitter and $-60^{\circ}$ in the receiver), making reference to the line of sight (LoS) path.

We can obtain the channel capacity, where the transmitters and receivers are $\mathrm{Nt}$ and $\mathrm{Nr}$ and the averaged signal-to-noise rate (SNR) is $\rho$ at each receiving antenna, with the following equation [18]:

$$
\mathrm{C}=\log _{2}\left(\operatorname{det}\left(I_{N_{r}}+\frac{\rho}{N_{t}} H H^{H}\right)\right) \quad \text { bits } / \mathrm{s} / \mathrm{Hz}
$$

where $I_{N r}$ is the $N r \times N r$ identity matrix, ( )H is the Hermitian transposition, and $H$ is the normalized channel transfer matrix.

We normalize the channel transfer matrix, G, so that the path loss component is removed. Thus, solely the relative variations for responses across all elements are revealed. This gives us an average signal-to-noise ratio that remains constant and is not dependent on receiver position. The normalization mentioned above can be undertaken using the Frobenius norm, thus [19]:

$$
H=\frac{G}{\sqrt{\frac{\sum_{j=1}^{N_{r}} \sum_{k=1}^{N_{t}}\left|G_{j, k}\right|^{2}}{N_{t} N_{r}}}}
$$

With this method we calculated the CDF of capacity for Room 2, as shown in Figure 23, for both types of walls being examined, taking the frequency variation analysis illustrated in Figure 24 into account and using a variety of receiver/transmitter positions, as shown in Figure 26.

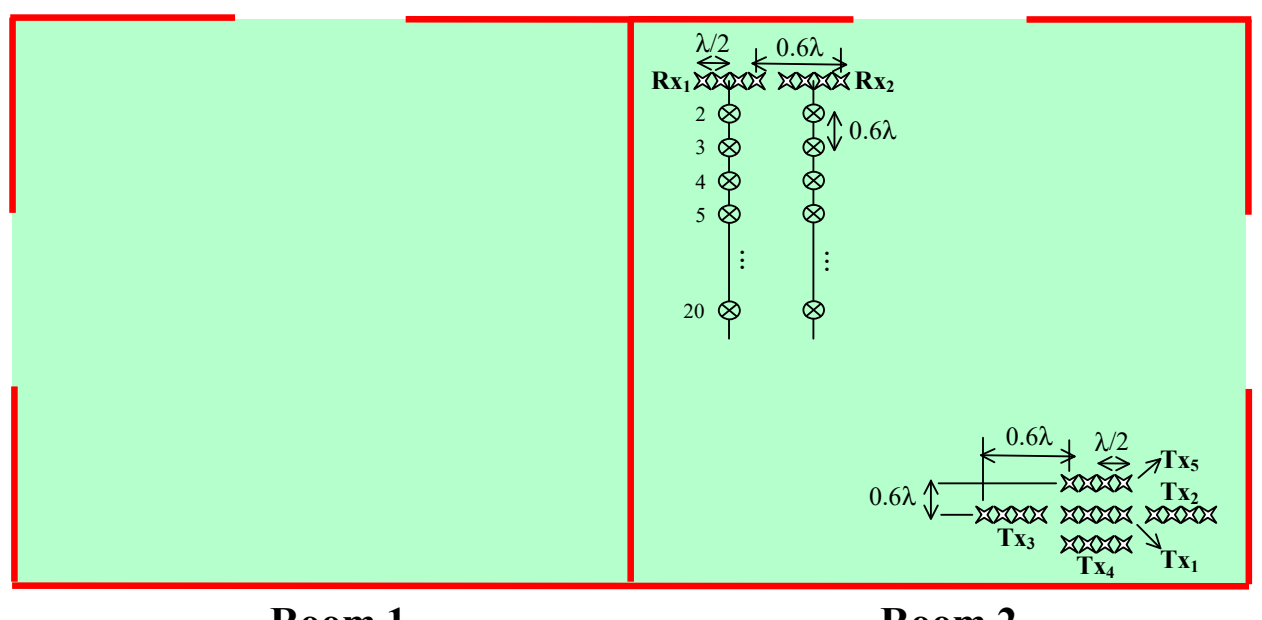

\section{Room 1}

Room 2

Figure 26. Geometry of the two rooms and the MIMO transmitter and receiver locations (top view).

As shown, we assumed five unique transmitter positions, each $0.6 \lambda$ apart $(\mathrm{T} \times 1$ to $\mathrm{T} \times 5)$, and each comprising an array of four omnidirectional antennae with $\lambda / 2$ spacing. For each position, we considered a pair of linear paths, evaluating 20 unique receiver positions with $0.6 \lambda$ spacing (comprising arrays of four omnidirectional antennas with $\lambda / 2$ spacing). We assumed that both the transmitter and receiver are at the same locations as shown in Figure 23. The simulation parameters considered in the ray-launching code were the following:

- Omnidirectional antennas

- Frequency: $5 \mathrm{GHz}$

- Hard Polarization

- Transmitting and receiving antennas bearing a $1 \mathrm{~m}$ height

- Number of rays launched: 7200

- Number of considered reflections: 9 
- Number of considered reflections of diffracted rays: 4

- Number of considered transmissions: 4

- Number of considered transmissions of diffracted rays: 3 .

Figure 27 shows the previously mentioned CDF of capacity for Room 2 with both papered and un-papered walls with an SNR of $10 \mathrm{~dB}$. The plot also incorporates the CDF of the capacity for an independent and identically distributed (I.I.D) Rayleigh fading channel for comparison purposes.

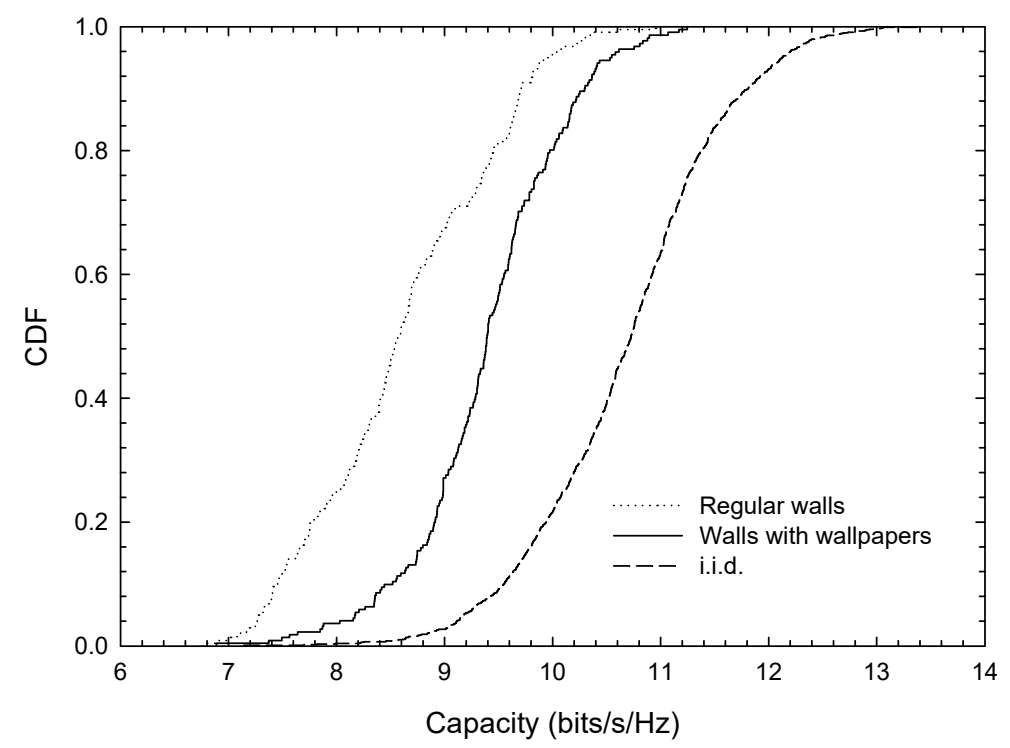

Figure 27. CDF of capacity in Room 2 for regular gypsum and F-S wall cases.

We can see that employing the F-S wallpaper leads to improved MIMO capacity; in particular, the capacity's mean value, for unpapered walls, is $8.6 \mathrm{bits} / \mathrm{s} / \mathrm{Hz}$; when the wall is papered, this rises to $9.4 \mathrm{bit} / \mathrm{s} / \mathrm{Hz}$.

In Figures 28 and 29, the calculated singular values (lambda 1 to 4 ) for the different transmitter and receiver positions are shown for the two types of walls under study. As can be observed, the additional degree of freedom previously seen in Figure 24 in the wallpaper case with the study performed as a function of frequency is also obtained for all the transmitter and receiver locations, which means that the improvement in capacity due to the use of the designed wallpapers does not depend on the position of the transmitter and receiver.

The above-mentioned advantage can also be noted in Table 1, where the mean and standard deviation of the four singular values calculated for all considered positions are shown, for both regular (common) gypsum and frequency selective wall cases.

Table 1. Mean and standard deviation of the singular values (lambda 1 to 4 ) obtained for all transmitter and receiver positions of Figure 26.

\begin{tabular}{cccc}
\hline & & Mean & Standard Deviation \\
\hline Regular & Lambda1 & 1.5254 & 0.50602 \\
Walls & Lambda2 & 0.63897 & 0.14742 \\
& Lambda3 & 0.17615 & 0.064675 \\
& Lambda4 & 0.012362 & 0.0081471 \\
\hline \multirow{2}{*}{ Walls with } & Lambda1 & 2.9068 & 0.68067 \\
Wallpaper & Lambda2 & 1.5055 & 0.49311 \\
& Lambda3 & 0.53912 & 0.18192 \\
& Lambda4 & 0.19102 & 0.061508 \\
\hline
\end{tabular}




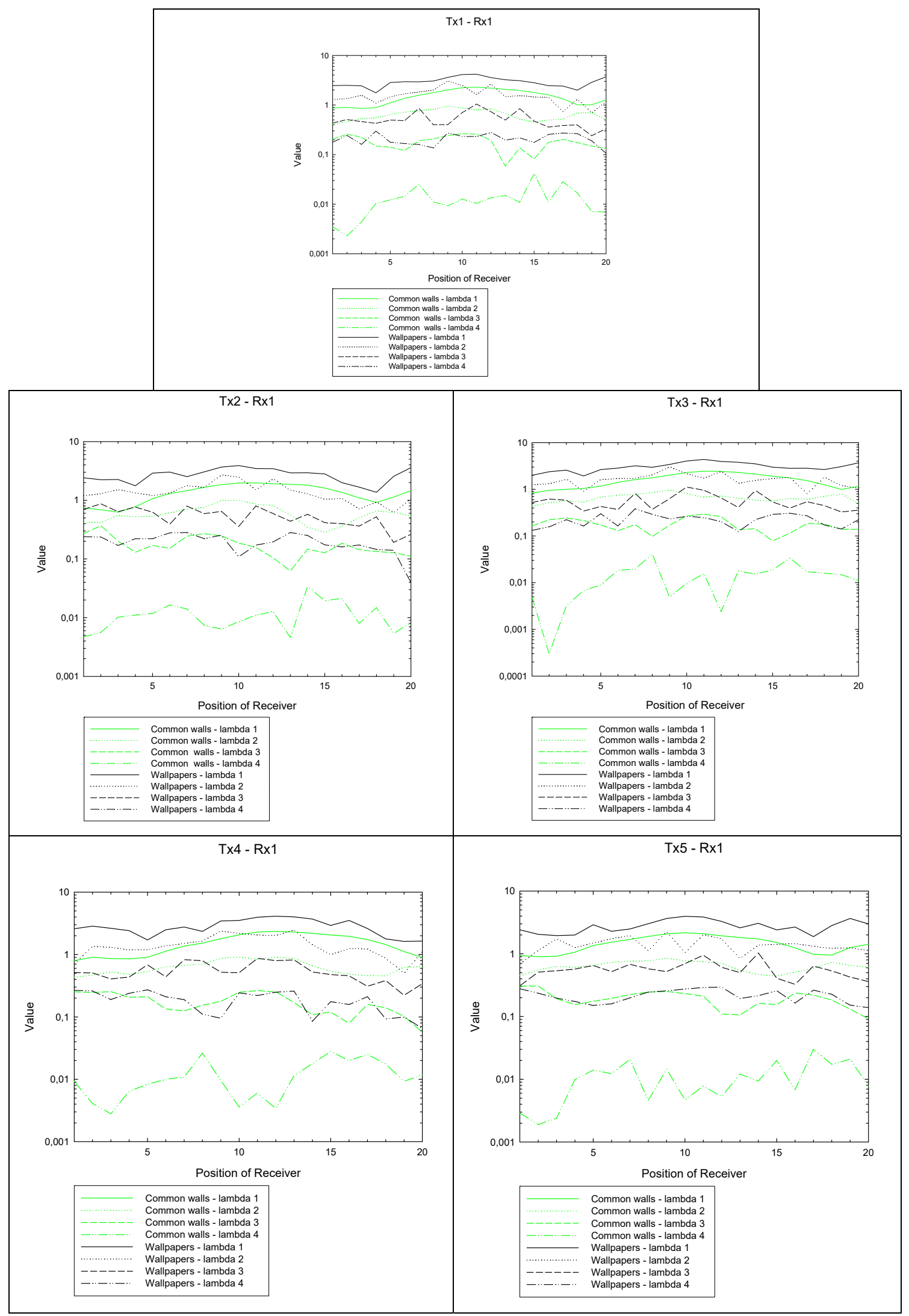

Figure 28. Singular values for the channel transfer matrix considering the geometry of Figure 26 (I). 


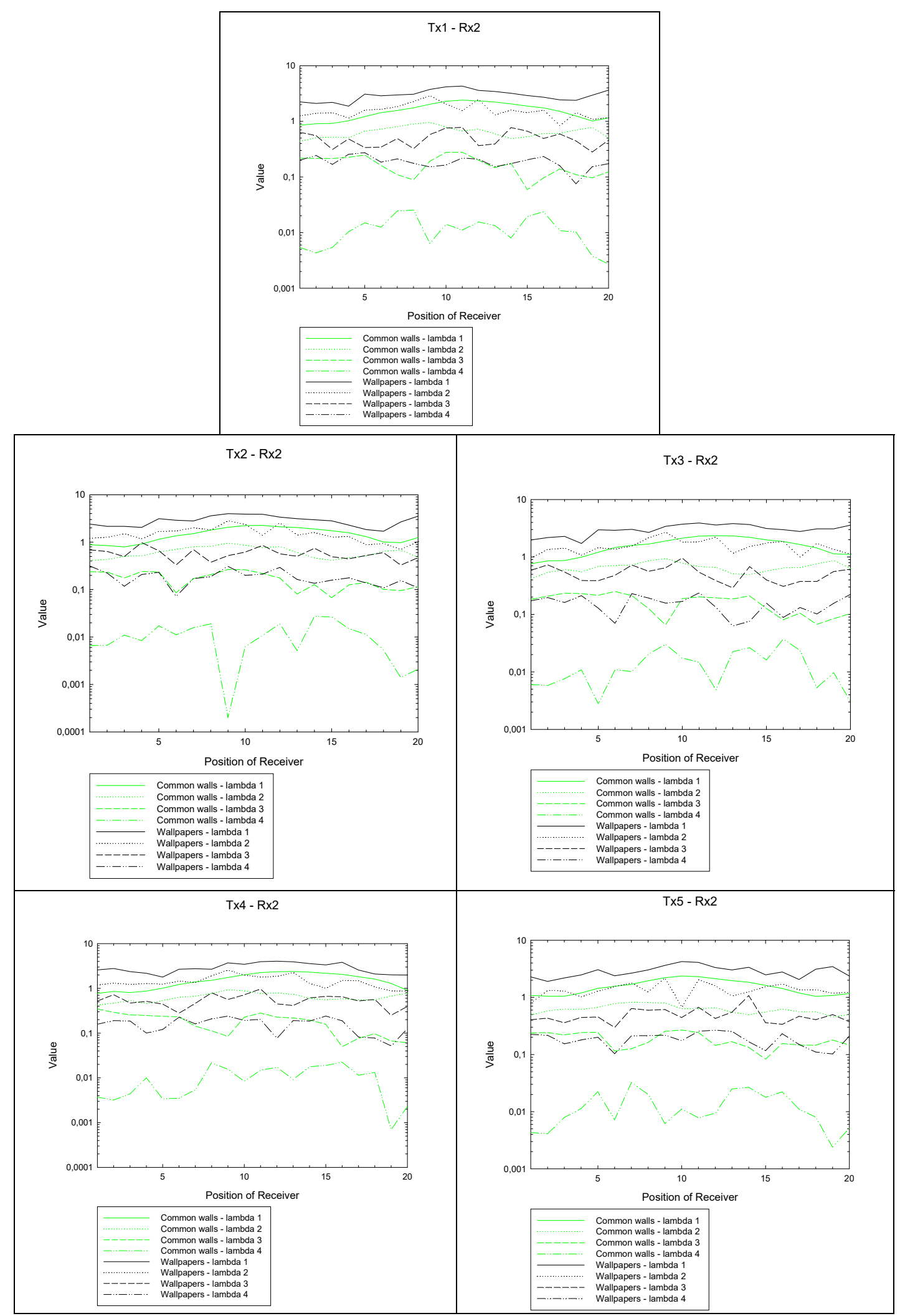

Figure 29. Singular values for the channel transfer matrix considering the geometry of Figure 26 (II).

\section{Conclusions}

F-S wallpaper has been designed with the intention of being mounted on standard walls to allow filtering of signals operating at $5 \mathrm{GHz}$ while simultaneously permitting required radio communication 
services on other frequencies to be received. The outcomes of this experiment unequivocally demonstrated that using this wallpaper in an indoor scenario reduces interference levels by an additional attenuation of $20 \mathrm{~dB}$ in comparison to an unpapered wall. Additionally, with MIMO systems, radio channel characteristics, such as capacity, are improved due to the increase in the magnitude of all singular values of the channel transfer matrix compared to the unpapered wall case, thereby leading to the existence of more relevant subchannels.

Author Contributions: Conceptualization, J.-V.R., M.G. and I.R.-R; methodology, J.-V.R., M.G. and J.-M.M.-G.-P.; software, J.-M.M.-G.-P., M.G. and L.J.-L.; validation, J.-V.R., J.-M.M.-G.-P. and M.G.; formal analysis, J.-V.R., M.G. J.-M.M.-G.-P. and I.R.-R.; investigation, J.-V.R., M.G. J.-M.M.-G.-P., L.J.-L. and I.R.-R.; resources, J.-V.R., M.G. and I.R.-R.; data curation, J.-V.R., M.G., I.R.-R. and J.-M.M.-G.-P.; writing-original draft preparation, J.-V.R.; writing-review and editing, J.-V.R., M.G. and J.-M.M.-G.-P.; visualization, J.-V.R., M.G.; supervision, J.-V.R., M.G.; project administration, J.-V.R. and J.-M.M.-G.-P.; funding acquisition, J.-V.R. and J.-M.M.-G.-P. All authors have read and agreed to the published version of the manuscript

Funding: This work has been funded by the Ministerio de Economía y Competitividad (MINECO), Spain (TEC201678028-C3-2-P), and by European Fonds Européen de Développement Économique et Régional (FEDER) funds.

Acknowledgments: The financial support by the Swedish Research Council is gratefully acknowledged. José-Víctor Rodríguez would like to thank the Universidad Politécnica de Cartagena (UPCT), Spain, for the grant received within the framework of the Training and Mobility Programme for Researchers and Professors (PMPDI-UPCT-2006) of the UPCT for the stay at Lund University, Sweden. Ignacio Rodríguez-Rodríguez would like to thank the support of Programa Operativo FEDER Andalucía 2014-2020 under Project No. UMA18-FEDERJA-023 and Universidad de Málaga, Campus de Excelencia Internacional Andalucía Tech.

Conflicts of Interest: The authors declare no conflict of interest. The funders had no role in the design of the study; in the collection, analyses, or interpretation of data; in the writing of the manuscript; or in the decision to publish the results.

\section{References}

1. Niembro-Martin, A.; De Barros, F.; Eymin-Petot-Tourtollet, G.; Lemaitre-Auger, P.; Pistono, E.; Vuong, T.-P. Metapaper: A frequency selective surface wallpaper for the attenuation of Wi-Fi signals. Eur. Microw. Conf. (EuMC) 2015. [CrossRef]

2. Sideeq, M.M.; Qasem, N. Smart Wall Based on Active Frequency Selective Wallpaper. In Proceedings of the 1st International Conference on Engineering and Innovative Technology, SU-ICEIT 2016, Salahaddin University-Erbil, Kurdistan, Iraq, 12-14 April 2016.

3. Qasem, N.; Aldorgam, E.; Alzou'bi, H. Overcoming the Influence of Human Shadowing and Obstacles via Modified Building Using Frequency Selective Wallpapers for 60 GHz. J. Commun. Comput. 2016, 13, 90-101. [CrossRef]

4. Cho, S.; Hong, I. Reduction of wireless signals in indoor environments by using an active frequency selective wall based on spectrum sensing. Int. J. Commun. Syst. 2017, 30, e3370. [CrossRef]

5. Döken, B.; Kartal, M. Easily Optimizable Dual-Band Frequency-Selective Surface Design. IEEE Antennas Wirel. Propag. Lett. 2017, 16, 2979-2982. [CrossRef]

6. Yin, W.; Zhang, H.; Zhong, T.; Min, X. A Novel Compact Dual-Band Frequency Selective Surface for GSM Shielding by Utilizing a 2.5-Dimensional Structure. IEEE Trans. Electromag. Compat. 2018, 60, 2057-2060. [CrossRef]

7. Cho, S.; Lee, I.; Hong, I. Hong. Frequency Selective Film Design for Building Walls for Blocking Wireless LAN Signal. In Proceedings of the 2018 International Symposium on Antennas and Propagation (ISAP), Busan, Korea (South), 23-26 October 2018; pp. 1-2.

8. Sung, G.H.; Sowerby, K.W.; Neve, M.J.; Williamson, A.G. A Frequency-Selective Wall for Interference Reduction in Wireless Indoor Environments. IEEE Antennas Propag. Mag. 2006, 48, 29-37. [CrossRef]

9. Sung, G.H.; Sowerby, K.W.; Williamson, A.G. Modeling a Low-Cost Frequency-Selective Wall for WirelessFriendly Indoor Environments. IEEE Antennas Wirel. Propag. Lett. 2006, 5, 311-314. [CrossRef]

10. Telatar, I.E. Capacity of Multi-Antenna Gaussian Channel. Eur. Trans. Telecommun. 1999, 10, 585-595. [CrossRef]

11. Gustafsson, M.; Karlsson, A.; Pontes Rebelo, A.P.; Widenberg, B. Design of Frequency Selective Windows for Improved Indoor Outdoor Communication. IEEE Trans. Antennas Propag. 2006, 54, 1897-1900. [CrossRef]

12. Munk, B.A. Frequency Selective Surfaces: Theory and Design; Wiley: New York, NY, USA, 2000. 
13. Raspopoulos, M.; Stavrou, S. Frequency Selective Buildings Through Frequency Selective Surfaces. IEEE Trans. Antennas Propag. 2011, 59, 2998-3005. [CrossRef]

14. Balanis, C.A. Advanced Engineering Electromagnetics; John Wiley \& Sons, Inc.: New York, NY, USA, 2005; pp. 177-184.

15. Molina-Garcia-Pardo, J.-M.; Rodríguez, J.-V.; Juan-Llacer, L. Wide-band measurements and characterization at 2.1 GHz while entering in a small tunnel. IEEE Trans. Veh. Technol. 2004, 53, 1794-1799. [CrossRef]

16. Luebbers, R.J. Finite Conductivity Uniform GTD versus Knife Edge Diffraction in Prediction of Propagation Path Loss. IEEE Trans. Antennas Propag. 1984, 32, 70-76. [CrossRef]

17. Chuan, C.-N.; Kahn, J.M.; Tse, D. Capacity of Multi-Antenna Array Systems in Indoor Wireless Environments. In Proceedings of the IEEE Global Telecommunications Conference (GLOBECOM 98), Sydney, Australia, 8-12 November 1998.

18. Foschini, G.J.; Gans, J. On Limits of Wireless Communications in a Fading Environment when using Multiple Antennas. Wirel. Personal Commun. 1998, 6, 311-335. [CrossRef]

19. Molina-Garcia-Pardo, J.-M.; Rodríguez, J.-V.; Juan-Llacer, L. Parametric spherical wave multiple-input and multiple-output model for ray-based simulations. Radio Sci. 2007, 42. [CrossRef]

(C) 2020 by the authors. Licensee MDPI, Basel, Switzerland. This article is an open access article distributed under the terms and conditions of the Creative Commons Attribution (CC BY) license (http://creativecommons.org/licenses/by/4.0/). 